\title{
The Use of Blocked Isocyanates to Increase the Adhesion of the Glue-Free Rubber - Polyester Cord
}

\author{
Nelly N. SHISHKINA \\ Ph.D. (in Technical Sciences) \\ Associate Professor \\ Technology of Synthetic Rubber Department \\ Faculty of Rubber and Elastomer Technology and Processing \\ Kazan National Research Technological University \\ 68, Karl Marx Str., Kazan, 420015, Russia \\ shishkina.nelli@bk.ru \\ Larisa Yu. ZAKIROVA \\ Ph.D. (in Technical Sciences) \\ Associate Professor \\ Chemistry and Processing Technology of Elastomers Department \\ Faculty of Rubber and Elastomer Technology and Processing \\ Kazan National Research Technological University \\ 68, Karl Marx Str., Kazan, 420015, Russia \\ zakirova.knitu@mail.ru
}

\begin{abstract}
The strength of the bond between the cord and rubber has a huge impact on the quality of reinforced structural materials. There are two methods of increasing adhesion: chemical and physical modification of the cord itself and the introduction of special additives into the rubber matrix. The authors propose to use reactive compounds - isocyanates as an additive to increase adhesion to polyester cord. In view of the complexity and toxicity of the latter, the approach of blocking isocyanate groups and the further introduction of these systems into the polymer matrix was chosen. The blocked isocyanate - 2,4 diphenylcarbamidotoluene was synthesized; the structure was identified by IR and elemental analysis. The effect of the amount of the synthesized additive on the adhesion to the polyester cord was investigated. The introduction of 2.4 diphenylcarbamidotoluene in an amount of 2.5 mass \% in the standard rubber compound based on SKI-3 showed a slight increase in the adhesive strength of the rubber-polyester cord bond.
\end{abstract}

Keywords: 2,4-diphenylcarbamidotoluene; synthesis; identification; rubber compound; adhesion.

\section{Introduction}

A feature of rubber-textile products is that they are almost always created as structures and in most cases they are obtained by combining textile reinforcing filler and rubber blanks with subsequent vulcanization. Rubber textile materials and products represent a separate group of structural composites, in which the matrix - rubber is a limited crosslinked rubber with high deformability and low modulus of deformation. This is determined by the amorphous structure and low glass transition temperature of rubber - lower than the operating temperature (Perepelkin, 2009).

The manufacture of rubber textiles began with the invention (patent) of Charles McIntosh, registered in 1823 - to use rubberized fabric for the manufacture of raincoats. For many years, such 
raincoats have been called "mackintosh" after the name of the inventor. A huge impetus for the development of rubber-textile products - rubber goods - was the emergence of self-propelled carriages - cars and the invention of pneumatic tires, which today are the main type of highly responsible rubber-textile products. Hoses and V-belts, airbags, and many other parts are rubbertextile products, which no car and other means of transportation can do without.

Generally, rubber-textile products operate under conditions of predominant influence of tensile loads; they are easily deformed also when subjected to bending or compressive loads. In rubbertextile materials, the main structural element are threads or systems of threads. Filaments can be composed of fibers (yarns) or continuous man-made filaments. Their constituent fibers or filaments are combined into a single structural element by compulsory twisting and impregnated with a binder rubber component. The most important condition for the reinforcement of rubber-textile materials and products, as already mentioned, is the low value of the deformation modulus of the matrix (rubber) in comparison with the threads Em $\leq$ En (Perepelkin, 2009).

In contrast to structural composites, where the reinforcing fibrous filler is usually located evenly throughout the entire volume of the composite (and in many cases is oriented in the direction of the acting mechanical loads), rubber-textile products are specially designed structures with a given arrangement of fibrous filler in the direction of tensile loads and rubber layers between them. Due to the low modulus of deformation and high deformability, the layers of rubber hardly impede bending and compressive loads. Today, rubber-textile products are a wide class of the most necessary products in our life and technology. They include a large number of different types, including the following:

- automobile, aviation and other types of tires;

- transfer elements of devices for moving various materials (conveyor belts, sleeves, hoses);

- flexible traction links of gears (drive belts, caterpillar belts, etc.);

- inflatable products and structures;

- human protective equipment (suits, aprons) and many others.

\section{Reinforcing Fibrous Fillers (Industrial Yarns, Belting, Fabrics)}

Industrial yarns, belting, cord fabrics are textile materials used for the manufacture (reinforcement) of automobile and aircraft tires, as well as rubber-textile products (conveyor belts, drive belts, hoses, and others). The main types of industrial yarns used as initial ones for reinforcing textile structures are viscose (currently they are not widely used), aliphatic polyamide (polyamide 6 nylon and polyamide 66 -anide) polyester. For heavily loaded tires, parapolyamide threads are used. For special types of tires, carbon, glass and metal threads are sometimes used. For some types of tires and other rubber-textile products, hydrated cellulose (viscose) industrial threads are still traditionally used. The properties of the listed types of industrial threads were briefly discussed above.

In very rare cases, cotton threads (yarns) are still used. The quality of the reinforcing threads (cord) is determined by the thickness and twist, its mechanical, thermal and chemical properties. For cord threads, a high level of their mechanical properties is especially necessary, since it is the threads that absorb the static and dynamic loads applied to the product. The modes of their testing are selected in accordance with the conditions of the deformation and stress state of the threads in the products that arise during their operation, often at elevated temperatures.

As already mentioned above, in contrast to structural fibrous polymer composites, where individual fibers surrounded by a matrix (binder) layer serve as a reinforcing element, in rubber-textile products, the main reinforcing element is threads, which are monolithized during their pre-treatment with adhesives and impregnation with rubber latexes. In the product, the cord frame forms a monolithic structure with rubber. It is a load-bearing element that takes up almost all mechanical stress. Therefore, the used reinforcing (cord) threads must have high resistance to various 
mechanical influences. The main types of woven structures used in rubber-textile products are shown in Figure 1.
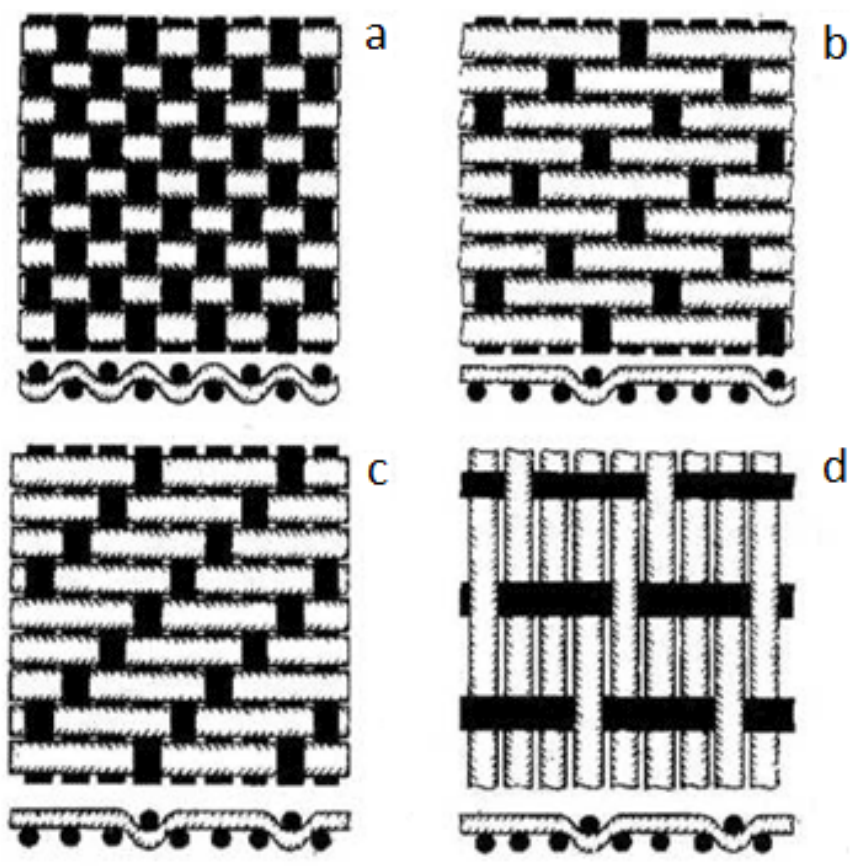

Figure 1. Schemes of the most common types of fabrics used to reinforce rubber-textile products: a - plain weave fabrics; $b$ - satin weave fabrics; $c$ - twill weave fabrics; $d$ - fabrics and ribbons with a sparsely spaced weft

Cord fabrics for the manufacture of tires are most often plain weave with a base of twisted cord threads of various linear densities (usually yarns in the range of linear densities of 125-500 tex are used) and a very rare weft of fine threads (about 15-25 tex). This design of the cord fabrics is due to the design of the tire carcass, in which mechanical stresses must act in the direction of the warp yarns. Thus, the performance properties of the cords in tires are determined by the properties of the cords. The weft is necessary so that the warp of the fabric does not fall apart during the tire making process. In the name of the cord fabric, the number indicates the rounded value of breaking load in $\mathrm{kgf}$, and the letter indicates the type of thread. The most common domestic brands of nylon cord are $12 \mathrm{~K}$ and $23 \mathrm{~K}$, anide $-13 \mathrm{~A}$ and $23 \mathrm{~A}$, viscose $-15 \mathrm{~B}, 17 \mathrm{~B}, 18 \mathrm{~B}$ and $22 \mathrm{~B}$.

The endurance of rubber-textile products under operating conditions is determined not only by their design and the complex of properties of the main elements - the reinforcing frame and rubber, but also by the strength of the bond between them. In the case of using chemical threads, a strong bond is formed only after special treatment with adhesives, while when using cotton cord, such processing is not required.

Especially high demands are placed on the threads used as elements of the tire cord carcass. To assess their properties, it is necessary to apply some special test methods. When the tire hits obstacles, high deformations and stresses arise in the threads. Therefore, it is necessary to determine the limits of the cord resistance to various single actions. When operating on flat road sections, the cords undergo small, repetitive loads, which makes it necessary to evaluate their fatigue characteristics. To assess the change in the size of tires during their operation, it is necessary to determine the relaxation characteristics of the cords, as well as their creep. When the tire rolls at the rubber-cord interface, shear and compression deformations occur, accompanied by a large heat generation and which can cause delamination of the carcass. Therefore, it is necessary to evaluate the adhesion of the cord to the rubber. 
The mechanical properties of rubber-textile products are determined by semi-cycle, single-cycle and multi-cycle tests (the cycle includes the stages of loading, unloading and "rest" of the sample). In semi-cycle tests, including only the loading stage, the absolute and relative strength, stress at break of reinforcing threads, their relative elongation and tensile modulus are determined, which is conventionally estimated as a load at a given small elongation or elongation at a given small load.

The endurance of the cords under multiple deformations under various conditions is determined using multi-cycle tests. This indicator is assessed by the number of loading cycles before the destruction of the sample or by the relative drop in strength after a given number of cycles. In highcycle tests, the threads are repeatedly subjected to various types of deformation: stretching, bending, impact with copra, compression and bending in rubber-textile samples. In addition, tests are carried out for the resistance to delamination of the rubber-textile system under shear and compression deformations, in which shear stresses arise at the rubber-thread interface. Thus, the adhesion of the reinforcing threads to rubber in the multiple loading mode is assessed.

The most important conditions for the normal operation of rubber-textile products, especially those subjected to prolonged repeated deformations, is the preservation of a long-term adhesive bond of reinforcing threads with rubber (Table 1) (Perepelkin, 2009).

Adhesive bonds in the cord-adhesive-rubber system are distinguished by the presence of two interfaces: adhesive-cord thread and adhesive-rubber. When these bonds are formed, a somewhat blurred interface between the components is formed and the ingredients of the rubber compound migrate from the rubber to the cord and from the cord to the adhesive. At the cord-adhesive interface, the bond is provided due to the flow of the adhesive between the elementary fibers, as well as a result of the formation of intermolecular physical or chemical interactions between the fibers and the active functional groups of the adhesive.

Table 1. Some properties of reinforcing cords

\begin{tabular}{lccc}
\hline Indicators & Viscose cord & Polyamide cord & Polyester cord \\
\hline Density, $\left[\mathrm{g} / \mathrm{cm}^{3]}\right.$ & 1.52 & 1.15 & 1.38 \\
\hline Strength, [MPa] & $540-780$ & $780-880$ & $920-960$ \\
\hline $\begin{array}{l}\text { Wet strength retention, [\%] } \\
\text { Retention of strength after } \\
\text { heating at } 200{ }^{\circ} \mathrm{C} \text { for 2 hours, } \\
{[\%]}\end{array}$ & $70-75$ & $85-75$ & $96-100$ \\
\hline
\end{tabular}

At the interface between adhesive and rubber, under the influence of pressure and temperature during rubberizing and vulcanization, intermolecular and chemical interactions occur between the functional groups of the adhesive, fiber and rubber ingredients. The introduction of special additives with active functional groups into rubbers (resorcinol-formaldehyde resins, sulfochlorinated polyethylene, etc.) leads to a significant increase in the bond strength of the system due to the formation of chemical bonds at the rubber-adhesive interface. The adhesive film, which exceeds rubber in terms of the deformation modulus (in the area of small deformations up to $100 \%$ ), serves as a "transition bridge" between a high-modulus cord and a low-modulus rubber, taking on part of the stresses arising in the operating rubber-cord system.

The most widely used adhesives are based on natural, styrene-butadiene, carboxylate and vinylpyridine latexes. Resorcinol-formaldehyde resins in the form of phenol alcohols or low molecular weight oligomers are mainly introduced as active additives in latex compositions. 
Sometimes other components are also added. Usually, adhesives of the following composition (in parts by weight) are used: latex - 100, resorcinol-formaldehyde resin - 10-25 (sometimes also carbon black - 20-40).

Synthetic resin adhesives find limited use. The main group of these adhesives is occupied by diisocyanates, which are highly reactive. However, the work with these compounds is difficult due to their high sensitivity to moisture and the need to use organic solvents. This problem was solved by the use of blocked isocyanates. In them, free isocyanate groups are released during the hightemperature treatment of tires during the vulcanization process and interact with other components of the adhesive compounds and cords.

Adhesives based on the polycondensation product of epichlorohydrin and metaphenylenediamine have also been used commercially. The impregnation of the cord should ensure that 4-8\% of the adhesive is applied to its surface. The impregnated cord is dried to remove moisture; in this case, further condensation of the resorcinol-formaldehyde resin occurs in the adhesive film. For different types of cords, the technological process and the formulation of the adhesives are different. The technological process of impregnating the cord with adhesives is a rather complicated operation. Therefore, the methods of fastening the cord to rubber, based on the creation of chemical bonds between untreated cord and rubbers containing special additives, are increasingly being used. Impregnations similar in composition are used in the case of other rubber-textile products operating in the mode of prolonged repeated deformations / loads, for example, drive belts.

In the production of cord fabric, the main threads are polyester, polyamide, viscose fibers. Viscose cord was historically the first type of chemical cord. Their dry mechanical properties and sufficient thermal stability during operation ensured the manufacture of tires with sufficiently highperformance characteristics in comparison with tires based on cotton cord. However, the lack of resistance to moisture (puncture) and some other disadvantages of the viscose cord led to the creation of a synthetic cord to the displacement of the first polyamide and polyester cord as a reinforcing carcass.

Polyamide cords are characterized by lower adhesion to rubbers than viscose cords due to lower polarity and higher hydrophobicity. For the impregnation of the polyamide cord, latex adhesives with a higher content of resorcinol-formaldehyde resin and a higher concentration of impregnating compounds (18-20\% instead of 11-15 for viscose cord) are used. The disadvantages of polyamide cord are creep under load, increased shrinkage at high temperatures.

Polyamide threads have a relatively low glass transition temperature, $45-50^{\circ} \mathrm{C}$. Therefore, they have a noticeable creep. As a result, products made with the use of a polyamide cord wear out (increase in size) during operation, which reduces their service life. So, when the car is parked for a long time, these types of tires are irreversibly deformed. In such cases, tires with polyamide cord must be unloaded, cars must be put on blocks. In this case, nylon cord turns out to be worse than anide cord (nylon 66). On the other hand, the polyamide cord works perfectly under conditions of repeated dynamic loading - under operating conditions.

One of the ways to increase the modulus of a polyamide cord and eliminate damage to products is to extract it at high temperatures. Therefore, the processing technology of this cord includes the stages of thermal stretching and subsequent thermal stabilization. The temperature at these stages for nylon and anide cords is $190-200$ and $220-240^{\circ} \mathrm{C}$, respectively, with a residence time in each of the zones from 20 to $60 \mathrm{~s}$. The thermal stretching tension is from 20 to $50 \mathrm{~N}$ ( 2 to $5 \mathrm{kgf}$ ) per thread, depending on the type of cord.

Polyester cord yarns are characterized by high performance characteristics similar to polyamide ones, and surpasses it in fatigue strength. The use of polyester threads provides increased wear and heat resistance of tires, dimensional stability and soft, quiet ride. A significant advantage of 
polyester cord over polyamide is that it can be used for radial tires. Polyester cord is especially promising in belted-bias construction of tires for passenger cars.

The disadvantage of polyester yarns is a large thermal shrinkage, therefore, for it, as well as for a polyamide cord, heat setting is required. In terms of the complex of properties, polyester cord threads are superior to polyamide cord threads.

Another disadvantage of polyester cord is its poor adhesion to rubber. This is due to the fact that the polyester cord, in contrast to the polyamide cord, does not contain reactive groups, therefore the adhesion of the rubber compound to it is low (Grishin, 2016; Chen, Schlarb, 2018; Li, Wan, Zhang, Cui, Zhao, 2017; Ilyazov, Dorozhkin, Vlasov, Muhutdinov, 2000). It cannot be treated with conventional latex-resorcinol-formaldehyde compounds without preliminary chemical modification of the fibers. Therefore, to achieve high adhesion, it is necessary to surface modification of the polyester cord, for example, by plasma (plasma technologies are currently widely used for cleaning and modifying various surfaces, which makes it possible to bond synthetic materials without glue, improves the adhesion properties of the surface (Shashok, 2008; Koltsov, Ashmarin, Isakov, Vinogradov, Chernova, Petrov, Kuzmin, 2012; Ivanova, Danilov, Kolyamshin, Ushmarin, Kol'tsov, Khasanov, 2015)), or to develop and use new adhesives.

Previously, solutions of isocyanates in organic solvents or blocked isocyanates in an aqueous medium were used to increase the adhesion of a polyester cord to rubber, but the adhesion achieved did not meet the requirements of the technology. Recently, impregnating compositions have been developed, with the use of which the required adhesion of the cord to rubber is achieved during impregnation on the existing equipment of tire factories.

Sufficient bond strength with rubbers can be achieved only when polyester cord is treated with isocyanate solutions or aqueous dispersions of blocked isocyanates. Sometimes after that, the polyester cord is still treated with latex adhesives containing resorcinol-formaldehyde resins. A significant improvement in the adhesion properties of the polyester cord is also achieved as a result of high-temperature $\left(220-240^{\circ} \mathrm{C}\right)$ treatment of the impregnated cord. A polyester cord with adhesives introduced into its composition is also produced, which does not require such complex processing.

Currently, the most promising are studies in the development of adhesion promoters for attaching rubber to polyester cord. There are known works related to the treatment of polyester cord with an impregnating composition based on carboxyl-containing latex CKD-1C and vinylpyridine latex VP107. The use of an impregnating compound based on vinylpyridine latex with a high content of resorcinol-formaldehyde resin provided the best bond strength between rubber and polyester cord (Kasperovich, Krotova, Potapov, Reznichenko, Shkodich, 2017).

A possible solution to this problem is the use of adhesives that are capable of reacting both with the surface of the thread and with rubber. Epoxy resins, isocyanates, phenol-formaldehyde resins and acrylate resins can be used as adhesives. For example, isocyanates form urethane compounds with rubber, and isocyanate groups react with cord polyesters (Grishin, 2016). The disadvantage of this solution is the introduction of additional stages of surface preparation and adhesive application in the technological process.

It is known that isocyanates are used as adhesion promoters (Shishkina, Zakirova, Samuilov, 2019). However, they are not convenient for practical use (hydrolysis in air, toxicity, etc.). Therefore, we used blocked isocyanates.

The aim of the work was to increase adhesion in the glueless system rubber - textile cord using a new adhesive - blocked 2,4-diphenyl carbamidotoluene isocyanate.

\section{Materials and Methods}


Experimenting. The synthesis of 2,4-diphenylcarbamidotoluene was carried out in the absence of a solvent, the molar ratio of 2,4-toluene diisocyanate: aniline was 1: 1 . The calculated amount of aniline was loaded into a round-bottom flask and 2,4-toluene diisocyanate was slowly added through a separatory funnel. To reduce heating (exothermic reaction), the round-bottom flask was placed in a cooling bath. After the end of the reaction, the product, which was a white powder, was recrystallized in toluene.

Analyzes. The IR spectra of the product were recorded on an Infalyum spectrometer, the melting point was determined by the capillary method, elemental analysis was performed on a Maestro GC 7820 gas chromatograph with a mass-selective detector, the sample injection temperature was $250^{\circ}$ $\mathrm{C}$, the carrier gas was helium.

4 samples of rubbers based on SKI-3 were obtained. Sample 1 is presented with a standard cobalt salt adhesion promoter - manobond. Other samples (2-4) were prepared by replacing the manobond with the synthesized 2,4-diphenyl-carbamidotoluene. The quantitative content of 2,4-diphenylcarbamidotoluene varied from 2 to 5 mass parts per 100 mass parts of rubber. Vulcanization with polyester cord was carried out for 40 minutes at a temperature of $150^{\circ} \mathrm{C}$. For the obtained samples, the bond strength between rubber and a single cord thread was determined (N-method, GOST 14863-69).

\section{Results and Discussion}

The purpose of this work was to create a modifying additive 2,4-diphenyl-carbamidotoluene and use it in a rubber composition based on SKI-3 (synthetic isoprene rubber) as a promoter of rubber adhesion to a polyester cord. The synthesis of 2,4-diphenylcarbamidotoluene was carried out according to the known method described by Hennig:

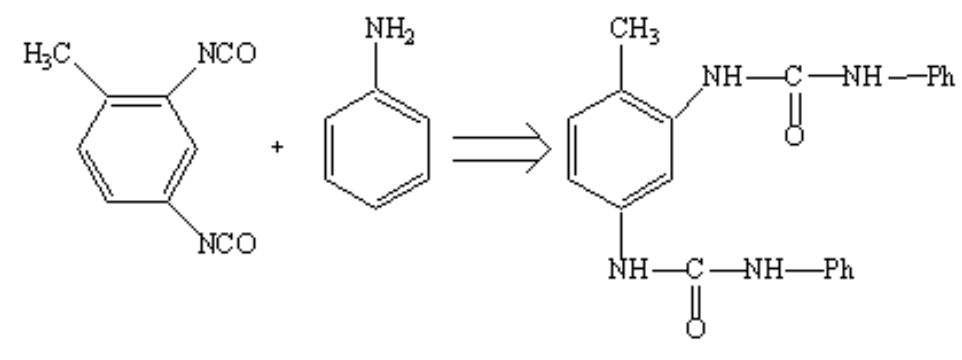

In the IR spectrum of 2,4-diphenylcarbamido-toluene, stretching vibrations of aromatic $\mathrm{C}-\mathrm{H}$ (3052 $\mathrm{cm}^{-1}$ ), stretching vibrations of the $\mathrm{C}-\mathrm{C}$ bond of the aromatic ring at $1617,1550,1501,1455 \mathrm{~cm}^{-1}$ are identified (Figure 2a). In the same place, in the region $\left(1650 \mathrm{~cm}^{-1}\right)$, stretching vibrations of the carbonyl group are identified $\mathrm{C}=\mathrm{O}$.

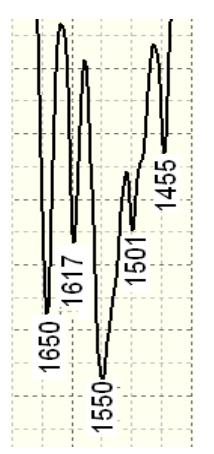

a)

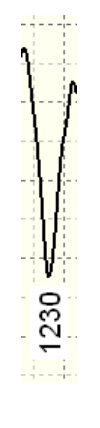

b)

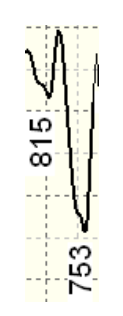

c)

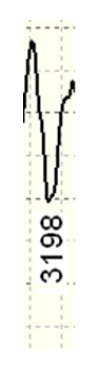

d)

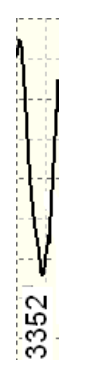

e)

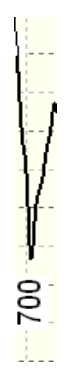

f) 
Figure 2. Fragments of the IR spectra of 2,4-diphenylcarbamidotoluene

Stretching vibrations of the carbon - oxygen bond correspond to the absorption band at $1230 \mathrm{~cm}^{-1}$ (Figure $2 \mathrm{~b}$ ). The spectrum also contains out-of-plane $\mathrm{C}-\mathrm{H}$ bending vibrations $\left(815,753 \mathrm{~cm}^{-1}\right)$ The spectrum also contains out-of-plane $\mathrm{C}-\mathrm{H}$ bending vibrations $\left(815,753 \mathrm{~cm}^{-1}\right)$ of the aromatic ring (Figure 2c).

Stretching vibrations of the $\mathrm{N}-\mathrm{H}$ group, the so-called amide 1, linked by hydrogen bonds, are identified in the region: asymmetric 3352 and symmetric $3198 \mathrm{~cm}^{-1}$ (Figure $2 \mathrm{~d}$, e). The spectrum overlaps a region of $1607 \mathrm{~cm}^{-1}$, which corresponds to the region of bending vibrations of the $\mathrm{N}-\mathrm{H}$ bond, however, in the region of low frequencies (700-600 $\mathrm{cm}^{-1}$ ), a broad absorption band is clearly identified, which corresponds to bending vibrations of the nitrogen-hydrogen bond $\left(700 \mathrm{~cm}^{-1}\right)$, (Figure 2e).

The elemental analysis results presented in Table 2 show that the deviation from the calculated results obtained is no more than $0.4 \%$. The melting point of 2,4-diphenylcarbamidotoluene is $144^{\circ} \mathrm{C}$, which coincides with the literature data. Thus, the totality of the results obtained makes it possible to identify the synthesized compound as 2,4-diphenylcarbamidotoluene.

Table 2. Elemental analysis of the synthesized 2,4-diphenylcarbamidotoluene

\begin{tabular}{lcccc}
\hline & \multicolumn{4}{c}{ Found [\%] } \\
\cline { 2 - 5 } & $\mathrm{C}$ & $\mathrm{H}$ & $\mathrm{N}$ & $\mathrm{O}$ \\
\hline Test 1 & 70.09 & 5.64 & 15.54 & 8.90 \\
\hline Test 2 & 70.15 & 5.56 & 15.50 & 8.85 \\
\hline Test 3 & 69.99 & 5.66 & 15.53 & 8.83 \\
\hline Average value & $70.08 \pm 0.01$ & $5.50 \pm 0.04$ & $15.52 \pm 0.09$ & $8.80 \pm 0.06$ \\
\hline Calculated & 70.00 & 5.55 & 15.55 & 8.88 \\
\hline
\end{tabular}

Next, the synthesized 2,4-diphenylcarbamidotoluene in various percentages (from 0.1 to $5 \mathrm{wt} \%$ ) was introduced into the standard rubber compound based on SKI-3. The adhesive strength of rubber with a cord was assessed by the N-method. The test results are shown in Table 3.

Table 3. Dependence of the bond strength of rubber-polyester cord on the content of 2,4diphenylcarbamidotoluene

\begin{tabular}{ccc}
\hline № & The amount of 2,4-diphenylcarbamidotoluene, [mass \%] & Bond strength rubber - cord, [N] \\
\hline 1 & 0.1 & 11 \\
\hline 2 & 1.0 & 15 \\
\hline 3 & 2.5 & 9 \\
\hline 4 & 5.0 & 11
\end{tabular}

The results indicate that blocked isocyanates, in particular 2,4-diphenylcarbamidotoluene, are capable of increasing the adhesion of rubbers to polyester cord. The maximum value of the bond strength of the rubber-polyester cord was only $15 \mathrm{MPa}$, which is only $30 \%$ higher than the adhesion performance of the polyester cord without additives.

This may be due to the fact that during the vulcanization process, when the temperature rises to $150{ }^{\circ} \mathrm{C}$, decomposition of 2,4-diphenylcarbamidotoluene can occur in two directions (Pizzi, Mettal, 2018): with 
the formation of aniline and isocyanate (direction 1), and direction 2 with the formation of aniline is obviously predominant, i.e., no active groups are formed that can form strong chemical bonds with the surface of the polyester cord:

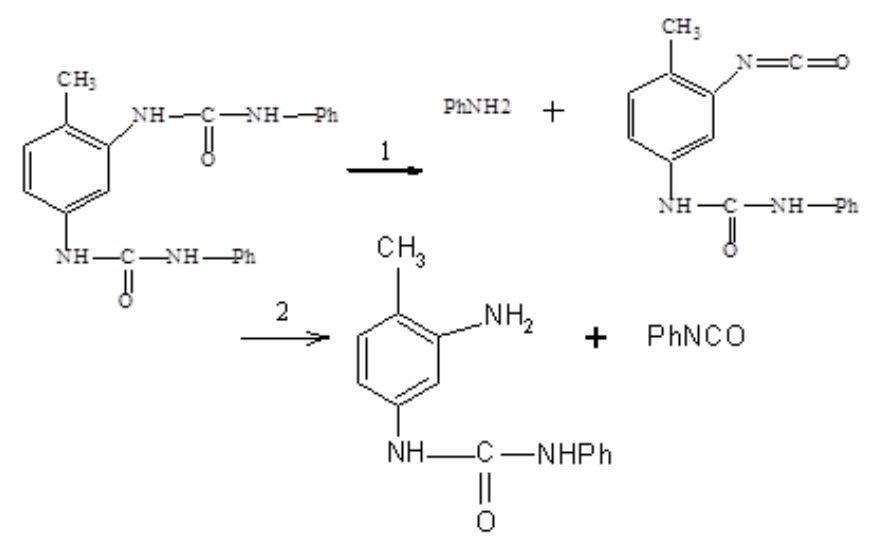

\section{Conclusion}

Thus, an overview of modern approaches to the adhesion of rubbers and polymer cords is presented. Studies have shown the possibility of using blocked isocyanate-2,4-diphenylcarbamidotoluene as an additive that increases the adhesion of the adhesive-free rubber-polyester cord. However, the achieved adhesion characteristics are insufficient for practical application and further research in this area is required.

\section{References}

Chen, Y., Schlarb, J.L. (2018). Steel cord-rubber adhesion with SEM/EDX. Tire Science and Technology, 46(1), 27-37.

Grishin, B.S. (2016). Theory and practice of elastomers strengthening. Kazan: KNITU. (in Russian)

Ilyazov, R.S., Dorozhkin, V.P., Vlasov, G.Ya., Muhutdinov, A.A. (2000). Tires. Operation and production problems. Kazan: KNITU.

Ivanova, A.V., Danilov, V.A., Kolyamshin, O.A., Ushmarin, N.F., Kol'tsov, N.I., Khasanov, A.I. (2015). The effect of maleinimides on the properties of a rubber compound based on nitrile butadiene rubber. International Polymer Science and Technology, 42(3), T19-T22.

Kasperovich, A.V., Krotova, O.A., Potapov, E.E., Reznichenko, S.V., Shkodich, V.F. (2017). A study of the influence of a new promoter on the adhesion of rubber to a metal cord. Polymer Science. Series, 9(1), 68-71.

Koltsov, N.I., Ashmarin, N.F., Isakov, S.A., Vinogradov, S.S., Chernova, N.A., Petrov, N.N., Kuzmin, M.V. (2012). Research of influence of technological additives on properties of rubbers based on BNR of new generation. Part 6. Combinations of softeners with fillers for increasing rubber frost resistance. Butlerov Communications, 29(3), 86-91.

Li, Z., Wan, J., Zhang, L., Cui, J., Zhao, S. (2017). Effects of heat and moisture on characteristics, tensile properties of RFL-coated rayon cords, and their adhesion with NR/SBR matrix. Journal of Applied Polymer Science, 134(48), 24-36.

Perepelkin, K.E. (2009). Reinforcing fibers and fibrous polymer compositions. Sankt-Peterburg: NOT. (in Russian)

Pizzi, A., Mettal, K.L. (2018). Handbook of Adhesive Technology. CRC Press. Taylor \& Francis Group, Lcc. 
Shashok, Zh.S. (2008). Problems of using domestic polyester cord in tire production BGTU. Series 2: Chemical Technologies, biotechnology, geotechnology, 103-106.

Shishkina, N.N., Zakirova, L.Yu., Samuilov, Ya.D. (2019). The effect of adhesive additives on the properties of isoprene rubber. Key Engineering Materials, 816, 255-259. 